〔Med. Entomol. Zool. Vol. 57 No. 3 p. 229-233 2006)

\title{
A new species of Simulium (Gomphostilbia) (Diptera: Simuliidae) from northern Thailand
}

\author{
Hiroyuki TAKAOKA ${ }^{1)}$ and Wej CHоoснотЕ ${ }^{2)}$ \\ 1) Department of Infectious Disease Control, Faculty of Medicine, Oita University, \\ Hasama, Yufu, Oita, 879-5593 Japan \\ 2) Department of Parasitology, Faculty of Medicine, Chiang Mai University, \\ Chiang Mai, 50200 Thailand
}

(Received: 29 May 2006; Accepted: 4 July 2006)

\begin{abstract}
A new simuliid species, Simulium (Gomphostilbia) udomi sp. nov., is described on the basis of the male reared from a pupa collected from northern Thailand. This new species is assigned to the ceylonicum species-group of the subgenus Simulium (Gomphostilbia), and is characterized in the male by the haired subcosta as well as the enlarged hind basitarsus, and in the pupa by the six gill filaments and the cocoon with an elongated anterodorsal projection.
\end{abstract}

Key words: black fly, Simuliidae, Simulium, Thailand, new species, ceylonicum speciesgroup

In Thailand, the ceylonicum speciesgroup of the subgenus Simulium (Gomphostilbia) Enderlein is represented by three species: $S$. (G.) asakoae Takaoka and Davies (Takaoka and Saito, 1996), S. (G.) inthanonense Takaoka and Suzuki (Takaoka and Suzuki, 1984) and S. (G.) sheilae Takaoka and Davies (Kuvangkadilok and Takaoka, 2000). During recent surveys on the immature stages of black flies in Nan Province, northern Thailand, we collected one more species of this species-group, which is characterized by the six pupal gill filaments in place of the usual eight filaments.

This is here described as a new species on the basis of the male adult (reared from a pupa), its associated pupal exuviae and cocoon.

The terms for morphological features used here follow those of Takaoka (2003). Holotype specimen of the new species is deposited at the Department of Infectious Disease Control, Faculty of Medicine, Oita University, Oita, Japan.

\section{Simulium (Gomphostilbia) udomi sp. nov.}

DESCRIPTION. Male. Body length $2.6 \mathrm{~mm}$. Head. Nearly as wide as thorax. Upper eye consisting of 13 vertical columns and 16 horizontal rows of large facets. Face dark brown, white pruinose. Clypeus dark brown, white pruinose, moderately covered with yellow simple long hairs interspersed with several dark brown simple long hairs on each side of lower portion. Antenna composed of $2+9 \mathrm{seg}$ ments, dark brown except scape, pedicel, and basal $1 / 3$ of 1 st flagellar segment yellow; 1 st flagellar segment elongate, about 1.7 times as long as 2nd flagellar segment. Maxillary palp composed of 5 segments on left side (Fig. 1A) but of 4 segments due to incomplete separation of 3 rd and 4 th segments on right side (Fig. 1B, C); proportional lengths of $3 \mathrm{rd}, 4$ th, and 5 th segments of left palp $1.0: 1.1: 2.7$; sensory vesicle of left palp (Fig. 1A) globular, with small opening, 0.17 times as long as 3rd segment; sensory vesicle of right palp globular and with large opening. Thorax. Scutum dark brown to brownish-black with anterolateral calli ocherous, shiny broadly along lateral margin and prescutellar area at certain angle of light, densely covered with yellowish scale-like re- 
Med. Entomol. Zool.
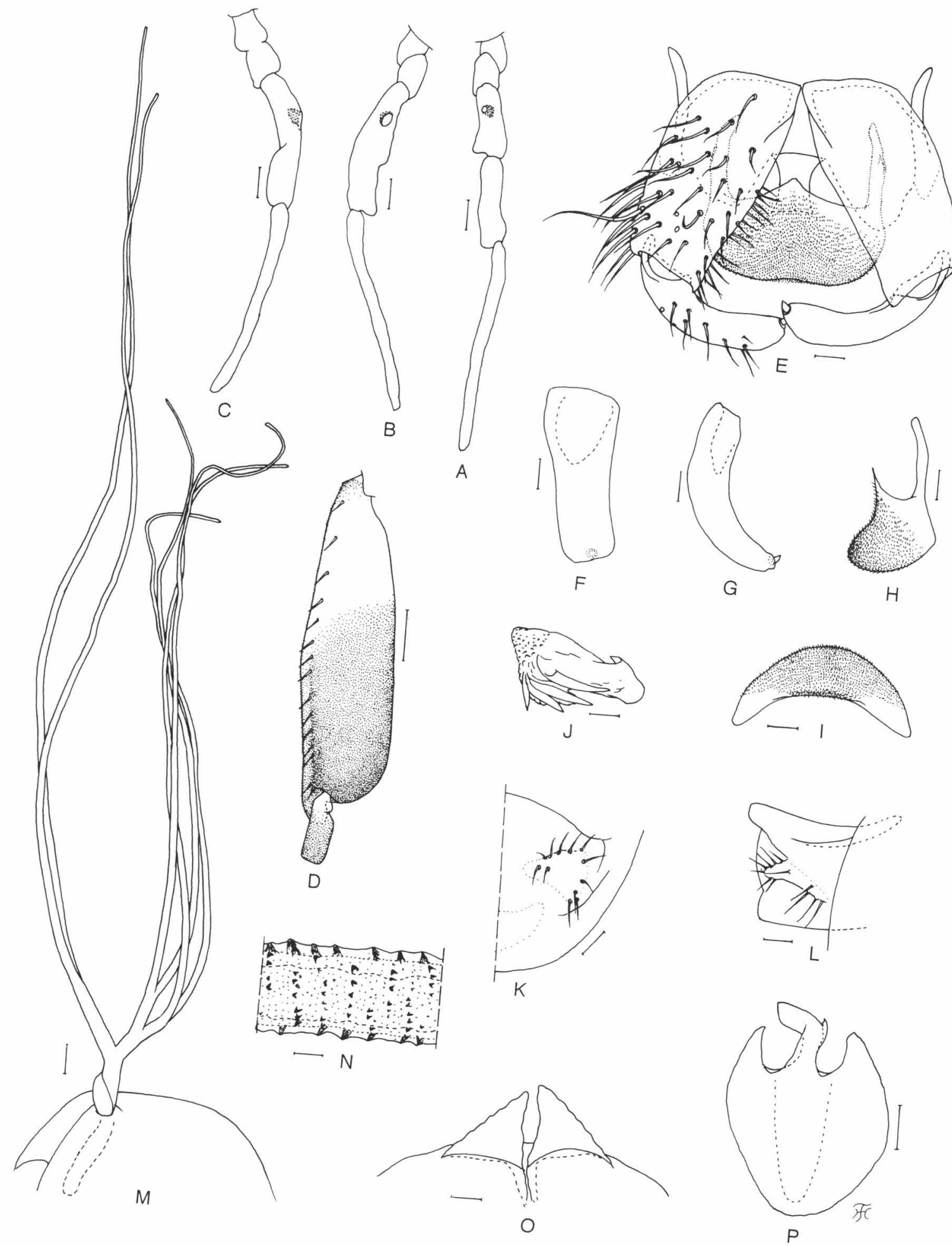
cumbent hairs (bright shiny when illuminated). Scutellum medium to dark brown, moderately covered with yellow short hairs and dark brown long upright hairs. Postnotum dark brown, slightly shiny at certain angle of light, thinly white pruinose, bare. Pleural membrane bare. Katepisternum dark brown, longer than deep, shiny at certain angle of light, moderately covered with yellow hairs as well as dark brown hairs. Legs. Foreleg: coxa yellow; trochanter yellow though posterior surface ocherous; femur medium brown; tibia yellowish-white basally and gradually darkened toward apex and brownish-black on apical $2 / 5$, though basal $3 / 5$ of outer surface yellowishwhite; tarsus brownish-black, with moderate dorsal hair crest; basitarsus slightly dilated, 8.0 times as long as its greatest width. Midleg: coxa medium brown though browinsh-black on posterior surface; trochanter dark yellow except base light yellow; femur medium brown though base of inner surface yellow; tibia brownish-black with basal $1 / 3$ yellow; tarsus brownish-black. Hind leg: coxa medium brown; trochanter yellow; femur medium brown with apical cap dark brown and extreme base yellow; tibia brownish-black with basal $2 / 5$ yellow; tarsus brownish-black except basal $2 / 5$ of basitarsus and basal $1 / 3$ of 2 nd tarsal segment yellowish-white; basitarsus (Fig. 1D) enlarged, wedge-shaped, gradually widened from base to middle, then nearly parallel-sided, 3.66 times as long as greatest width, and 1.0 and 1.26 times as wide as greatest widths of tibia and femur, respectively; calcipala small, slightly shorter than wide and 0.25 times as wide as greatest width of basitarsus. Wing. Length $2.4 \mathrm{~mm}$. Costa with dark spinules as well as dark hairs except basal batch of hairs mostly yellow. Subcosta with dark hairs except near apex bare. Hair tuft on stem vein yellow. Basal portion of radius fully covered with dark hairs. Basal cell absent. Abdomen. Basal scale brownish-black, with fringe of dark long hairs. Dorsal surface of abdomen dark brown to brownish-black except that of 2nd segment and anterior narrow portion of 3rd segment ocherous, moderately covered with dark hairs (though mixed with yellow hairs on anterior segments); segments 2, 5, 6 and 7 each with pair of shiny dorsolateral patches, those of segment 2 connected in middle to each other along anterior margin; ventral surface of abdomen dark brown except those of segments 2 and 3 yellowishwhite. Genitalia. Coxite in ventral view (Fig. $1 \mathrm{E})$ rectangular, about 2.1 times as long as wide. Style in posterolateral view (Fig. 1F) rectangular, about 2.4 times as long as its greatest width at base; style in medial view (Fig. 1G) 0.75 times as long as coxite, gently bent inward, with apical spine. Ventral plate in ventral view (Fig. 1E) transverse, much shorter than wide, with anterior margin sharply produced medially, and with posterior margin slightly convex medially, and densely covered with microsetae on ventral surface; basal arms of moderate length, slender, parallel-sided, though slightly converged apically; ventral plate in lateral view (Fig. $1 \mathrm{H}$ ) much produced ventrally; ventral plate in end view (Fig. 1I) rounded ventrally and densely covered with microsetae on posterior surface. Median sclerite (Fig. 1E) thin, plate-like, wide. Paramere (Fig. 1J) of moderate size, with 4 distinct stout hooks. Aedeagal membrane sparsely setose. Ventral surface of 10th abdominal segment without hairs near posterior margin on each side. Cercus in end view (Fig. $1 \mathrm{~K}$ ) triangular, with 12 or 13 hairs; cercus in lateral view (Fig. 1L) moderately produced posteriorly.

Pupa. Body length $3.5 \mathrm{~mm}$. Head. Integument yellowish-brown, moderately or somewhat sparsely covered with round tubercles; antennal sheath normal, with no spinous projections, and almost bare; face with pair of simple very long trichomes with coiled apex,

Fig. 1. Male and pupa of Simulium (Gomphostilbia) udomi sp. nov. A-L, male and M-P, pupa. A-C, maxillary palps with sensory vesicle (A, left side, front view; B, right side, front view; C, right side, lateral view); D, basitarsus and 2nd tarsal segment of hind leg (left side, outer view); E, coxites, styles, ventral plate and median sclerite in situ (ventral view); F and $\mathrm{G}$, styles ( $\mathrm{F}$, right side, ventrolateral view; $\mathrm{G}$, right side, medial view); $\mathrm{H}$ and $\mathrm{I}$, ventral plates $(\mathrm{H}$, lateral view; $\mathrm{I}$, end view); J, paramere (right side, end view); $K$ and $L$, 10th abdominal segments with cercus ( $K$, left side, end view; L, left side, lateral view); M, gill filaments (left side, lateral view); $\mathrm{N}$, part of middle portion of ventral pair of filaments; O, terminal hooks (posteroventral view); P, cocoon (dorsal view). Scale bars. $1.0 \mathrm{~mm}$ for P; 0.1 $\mathrm{mm}$ for $\mathrm{D}$ and $\mathrm{M} ; 0.04 \mathrm{~mm}$ for $\mathrm{A}-\mathrm{C} ; 0.02 \mathrm{~mm}$ for $\mathrm{E}-\mathrm{L} ; 0.01 \mathrm{~mm}$ for $\mathrm{N}$ and $\mathrm{O}$. 
and frons with 3 pairs of simple very long trichomes with coiled or uncoiled apex; 3 frontal trichomes on each side arising somewhat close together, subequal in length to one another, and slightly longer than facial ones. Thorax. Integument yellowish-brown, sparsely covered with round tubercles except posterodorsal surface moderately covered with round tubercles, with 3 pairs of simple very long trichomes with coiled apex anterodorsally, with 2 pairs of similar trichomes anterolaterally, with 1 pair of simple long trichomes with coiled apex posterolaterally, and with 3 pairs of simple trichomes (1 long with coiled apex, 1 medium-long with uncoiled apex and 1 short with uncoiled apex) ventrolaterally. Gill (Fig. 1M) composed of 6 slender filaments arranged in pairs, somewhat longer than pupal body, with somewhat swollen transparent organ ventrally at base; common basal stalk short, divided into upper short and lower medium-long primary stalks of equal thickness; lower primary stalk divided into 2 long filaments of equal size (3.6-3.8 mm long), while upper primary stalk divided further into upper and middle secondary stalks of short lengths, and each divided into 2 long filaments; upper and middle filaments subequal in length (2.3-2.6 mm long) and thickness; lower paired filaments slightly thicker than other 4 filaments; all filaments pale yellowish-brown, gradually tapered toward apical tip, with distinct annular ridges and furrows, and covered with relatively large dark brown tubercles on ridges and small ones on interridge spaces (Fig. 1N). Abdomen. Dorsally, all segments light yellowish-brown; segment 1 with 1 long simple slender seta on each side; segment 2 with 1 long simple slender seta and 5 short spinous setae on each side; segments 3 and 4 , each with 4 hooked spines and 1 short spinous seta on each side; segment 5 lacking spinecombs; segments 6-9 each with distinct spinecombs in transverse row, together with comblike groups of minute spines on each side; segment 9 with pair of terminal hooks closely located; terminal hook triangular in shape, plate-like, with weakly serrated outer margin (Fig. 10). Ventrally, segments $3-8$ with comb-like groups of minute spines; segment 4 with 2 medium-long simple hair-like setae and a few short simple slender setae on each side; segment 5 with pair of bifid hooks submedially and a few short simple slender setae on each side; segments 6 and 7 each with pair of bifid inner and simple outer hooks somewhat spaced from each other, and a few short simple slender setae on each side. Segment 9 with 3 grapnel-like hooklets on each lateral side. Cocoon (Fig. 1P). Wall-pocket-shaped, widely extending ventrolaterally, thinly woven appearing membrane; anterior margin very weakly defined having 1 or 2 small open spaces, and with very long and downwardly coiled anterodorsal projection; posterior $1 / 2$ with floor moderately woven; individual threads invisible; $4.0 \mathrm{~mm}$ long by 3.9 $\mathrm{mm}$ wide; anterodorsal projection, when extended, $2.3 \mathrm{~mm}$ long.

Female and Mature larva. Unknown.

TYPE SPECIMEN. Holotype male (with its associated pupal exuviae and cocoon), reared from a pupa collected from a small stream (width about $1.0 \mathrm{~m}$, depth about $0.5 \mathrm{~m}$, water temperature $17.0^{\circ} \mathrm{C}$, shaded, altitude $1,350 \mathrm{~m}$ ) moderately running in sparsely forested area, at Samorraphume, Thung Chang District, Nan Province, Thailand, 25. II. 2006, by W. Choochote.

ECOLOGICAL NOTES. The pupa of this new species was taken from trailing grass in the water. Associated species were S. (G.) asakokae, S. (G.) decuplum Takaoka and Davies, S. (Nevermannia) fruticosum Takaoka and Choochote, S. (Simulium) choochotei Takaoka, S. (S.) doipuiense Takaoka and Choochote, S. (S.) fenestratum Edwards and S. (S.) rudnicki Takaoka and Davies.

\section{DISTRIBUTION. Thailand.}

ETYMOLOGY. The species name $u d o m i$ is in honor of Dr. Udom Chaithong, Head, Department of Parasitology, Faculty of Medicine, Chiang Mai University, Chiang Mai, who has supported our works on Thai Simuliidae.

REMARKS. Simulium (G.) udomi sp. nov. is assigned to the ceylonicum speciesgroup of the subgenus Simulium (Gomphostilbia) because the male has an enlarged 
hind basitarsus (Fig. 1D). This new species is characterized by the pupal gill with six slender filaments (Fig. 1M), a character rarely occurring in the ceylonicum speciesgroup and so far found in the two species, i.e., $S$. (G.) rosemaryae Takaoka and Roberts described from Sulawesi (Takaoka and Roberts, 1988) and S. (G.) kamimurai Takaoka described from Sulawesi and Seram (Takaoka, 2003).

The male of $S$. (G.) udomi sp. nov. is easily separated from those of the latter two species in that it has a haired subcosta and a more slender fore basitarsus 8.0 times as long as its greatest width, while the latter two species have a bare subcosta and a fore basitarsus 6.5 times as long as its greatest width. In the pupal stage, this new species is also easily distinguished from the two known species by the cocoon with an elongated anterodorsal projection (Fig. 1P), the triangular terminal hooks (Fig. 1O) (cf., wide plate-like terminal hooks in the two known species) and the gill filaments covered with relatively large dark brown tubercles in transverse rows (Fig. 1N).

There is a distinct difference in the number of vertical columns of the large male eye facets between $S$. (G.) udomi sp. nov. and the three Thai known related species, i.e., the number of vertical columns is 13 in $S$. (G.) udomi sp. nov. while that is 11 in $S$. (G.) asakoae, 16 in $S$. (G.) inthanonense and 10 in $S$. (G.) sheilae (Takaoka and Davies, 1995; Takaoka and Suzuki, 1984).

\section{ACKNOWLEDGEMENTS}

This study was financially supported by a Grant-in-Aid for Oversea Research from the Japan Society for the Promotion of Science provided to HT (No. 18406011).

\section{REFERENCES}

Kuvangkadilok, C. and Takaoka, H. 2000. Taxonomic notes on Simuliidae (Diptera) from Thailand: Description of a new species and new distributional records of nine known species. Jpn. J. Trop. Med. Hyg., 28: 167-179.

Takaoka, H. 2003. The Black Flies (Diptera: Simuliidae) of Sulawesi, Maluku and Irian Jaya. xxii+581 pp., Kyushu University Press, Fukuoka.

Takaoka, H. and Davies, D. M. 1995. The Black Flies (Diptera: Simuliidae) of West Malaysia. viii +175 pp., Kyushu University Press, Fukuoka.

Takaoka, H. and Roberts, D. M. 1988. Notes on blackflies (Diptera: Simuliidae) from Sulawesi, Indonesia. Jpn. J. Trop. Med. Hyg., 16: 191-219.

Takaoka, H. and Saito, K. 1996. A new species and new records of black flies (Diptera: Simuliidae) from Thailand. Jpn. J. Trop. Med. Hyg., 24: 163-169.

Takaoka, H. and Suzuki, H. 1984. The blackflies (Diptera: Simuliidae) from Thailand. Jpn. J. Sanit. Zool., 35: 7-45. 